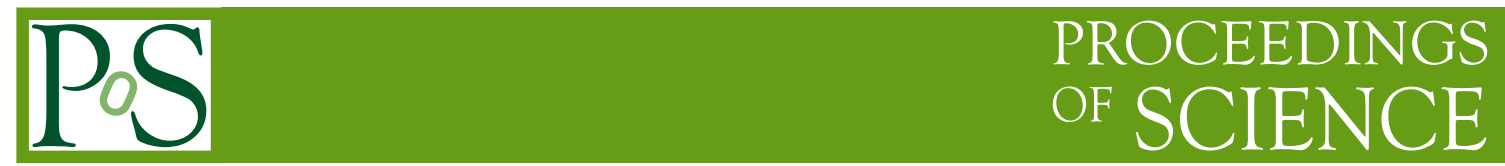

\title{
Vector-Boson Scattering at the LHC
}

\section{Christopher Schwan*}

Albert-Ludwigs-Universität Freiburg, Freiburg, Germany

E-mail: christopher.schwan@physik.uni-freiburg.de

We report on a calculation of the QCD NLO correction to the process $\mathrm{pp} \rightarrow \mathrm{e}^{+} v_{\mathrm{e}} \mu^{+} v_{\mu} \mathrm{jj}+\mathrm{X}$, which contains the like-sign $\mathrm{W}$-scattering process $\mathrm{W}^{+} \mathrm{W}^{+} \rightarrow \mathrm{W}^{+} \mathrm{W}^{+}$. We discuss the validity of the double-pole approximation in the context of this process and the $M_{\mathrm{H}} \rightarrow \infty$ limit, which allows one to study the unitarization effect of the standard model Higgs for different observables. Finally, we present a first calculation of the PDF uncertainties for this process.

13th International Symposium on Radiative Corrections 24-29 September, 2017

St. Gilgen, Austria

${ }^{*}$ Speaker. 


\section{Introduction}

Vector-boson scattering is a key class of processes to probe the Higgs couplings to massive vector-bosons and their (possibly anomalous) quartic gauge coupling. The effect of a single $125 \mathrm{GeV}$ standard model (SM) Higgs-boson is the unitarization of the cross section of the scattering of longitudinally polarized gauge bosons. This describes the fact that, without the Higgs-boson exchange, the cross section increases beyond any bound with increasing collision energy.

We discuss the process $\mathrm{pp} \rightarrow \mathrm{e}^{+} v_{\mathrm{e}} \mu^{+} v_{\mu} \mathrm{jj}+\mathrm{X}$ at LO and NLO QCD, which contains as subprocess the like-sign gauge-boson scattering $\mathrm{W}^{+} \mathrm{W}^{+} \rightarrow \mathrm{W}^{+} \mathrm{W}^{+}$. On the experimental side there is evidence [1] in ATLAS $8 \mathrm{TeV}$ data and an observation of CMS [2] with $13 \mathrm{TeV}$ data. Theoretical calculations for this process have been calculated at LO and NLO in QCD some time ago [3, 4], and recently there has been a publication of an all-channel NLO calculation [5], including the electroweak corrections which are quite large.

In this context we discuss the double-pole approximation and its validity, different scale choices, the first calculation of PDF uncertainties, and the limit $M_{\mathrm{H}} \rightarrow \infty$, which shows the effect of the absence of unitarization. The absence of a SM Higgs-boson can also be interpreted as the maximum effect of a different Higgs sector; any model with Higgs mixing will modify the SM Higgs coupling in such a way that the effective Higgs mass will be larger. We would like to stress that the Higgs mass is known and by sending it to infinity we would like to, in a gauge-invariant way, assess the effect of a different Higgs coupling.

In Sec. 2 we explain our setup and cuts, in Sec. 3 we explain the double-pole approximation (DPA), and in Sec. 4 we finally present numerical results.

\section{Setup}

We define the following fiducial phase-space volume, which is used to generate the results shown in Sec. 4 for the process $\mathrm{pp} \rightarrow \mathrm{e}^{+} v_{\mathrm{e}} \mu^{+} v_{\mu} \mathrm{jj}+\mathrm{X}$. The cuts are similar to the one defined for the ATLAS $8 \mathrm{TeV}$ analysis [1]:

- Jets are defined by the anti- $k_{\mathrm{T}}$ algorithm [6] with radius $R=0.4$ and a $p_{\mathrm{T}}>\mathrm{GeV}$ and rapidity of $|y|<4.5$. We require at least two jets, and call the two hardest jets tagging jets.

- Leptons pass the cut if $p_{\mathrm{T}}>27 \mathrm{GeV}$ and $|y|<2.5$. We require that there are exactly two leptons.

- The neutrinos are taken care of by requiring that the missing transverse energy is $E_{\mathrm{T}}>$ $30 \mathrm{GeV}$.

- We furthermore require that leptons are separated by each other by requiring $\Delta R>0.3$, and from the tagging jets by the same distance (but not from a possible third jet).

- The leptons must have an invariant mass of $M>20 \mathrm{GeV}$.

- To enhance the contribution of the signal process we furthermore require that the tagging jets have $M>500 \mathrm{GeV}$ and are separated in rapidity by $\left|y_{1}-y_{2}\right|>2.4$. The effect of these two cuts is illustrated in Fig. 1 where we use no approximations for the LO matrix elements. 
For the calculation of the matrix elements we employ an approximation that only uses the squares of $t$ - and $u$-channel diagrams and neglects $s$-channels and the remaining interferences; see Ref. [3] a definition of this approximation. For the virtual matrix elements we use a double-pole approximation, which is described in Sec. 3.

The parton distribution functions (PDF) used for this calculation are CT14LO for the leading order predictions and CT14NLO [7] for the NLO prediction. The scale used for both factorization and renormalization scale is

$$
\mu_{\mathrm{R}}=\mu_{\mathrm{F}}=\xi \mu=\xi \sqrt{p_{\mathrm{T}}^{2}\left(\mathrm{j}_{1}\right)+M_{\mathrm{W}}^{2}} .
$$

where $\xi=1$ for the central value; $p_{\mathrm{T}}\left(j_{1}\right)$ denotes the transverse momentum of the leading (tagging) jet. The theoretical uncertainties are estimated by the envelope of a three-point scale variation, i.e. by setting $\xi \in\{1 / 2,1,2\}$. The PDF uncertainties are estimated by using the Hessian method described in Ref. [8]. Please note that the PDF uncertainty presented here represents the $90 \%$ confidence level uncertainty that the CT14NLO PDF set by default represents.

All results were calculated using an in-house Monte Carlo developed by the author, which uses matrix elements from LUSIFER [9] and one-loop amplitudes from Stefan Dittmaier. The one-loop amplitudes make use the of COLLIER [10,11, 12, 13] library to evaluate one-loop integrals. The results were validated against a calculation done with SHERPA [14], which uses one-loop matrix elements from OpenLoops [15].
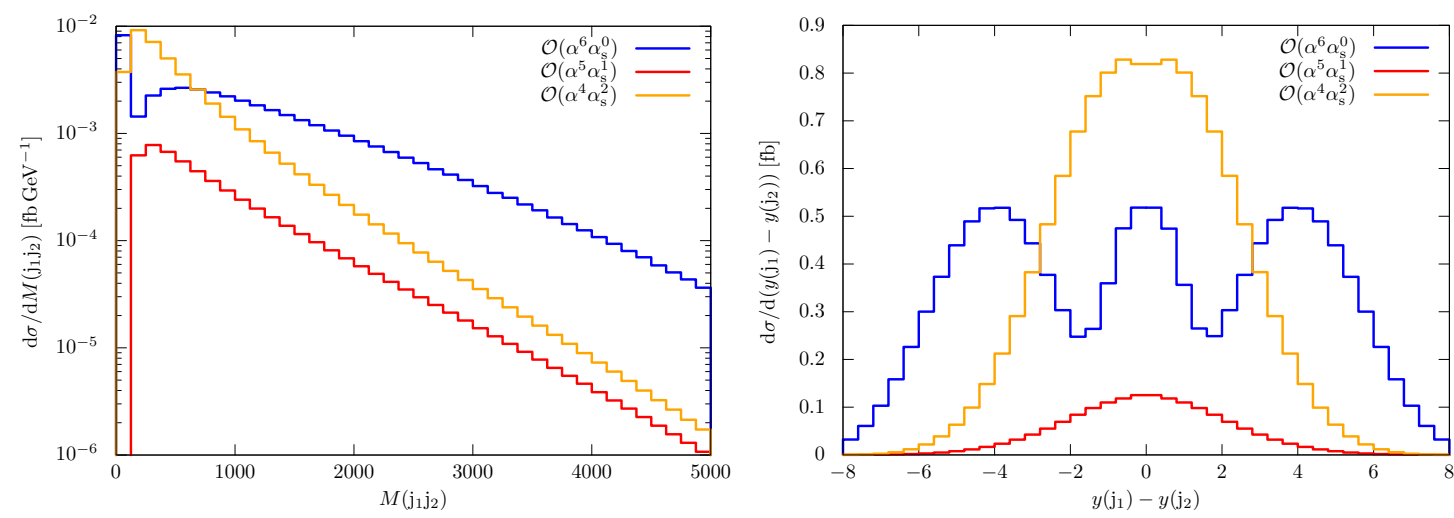

Figure 1: Leading-order predictions (no approximations used) for the process $\mathrm{pp} \rightarrow \mathrm{e}^{+} v_{\mathrm{e}} \mu^{+} v_{\mu} \mathrm{jj}+\mathrm{X} @$ $13 \mathrm{TeV}$ for an inclusive phase space, only requiring two anti- $k_{\mathrm{T}}$ jets with $p_{\mathrm{T}}>30 \mathrm{GeV}$. The phase space cuts on $M\left(\mathrm{j}_{1} \mathrm{j}_{2}\right)>500 \mathrm{GeV}$ and $\left|y\left(\mathrm{j}_{1}\right)-y\left(\mathrm{j}_{2}\right)\right|>2.4$ enhance the contribution $\mathscr{O}\left(\alpha^{6}\right)$ with respect to $\mathscr{O}\left(\alpha^{5} \alpha_{\mathrm{s}}\right)$ and $\mathscr{O}\left(\alpha^{4} \alpha_{\mathrm{s}}^{2}\right)$.

\section{Double-pole approximation}

The pole approximation (PA) is an expansion of an amplitude around its resonances. For this process we consider an expansion around the two resonant $\mathrm{W}^{+}$-bosons, which decay into charged 
leptons and neutrinos. Pictorially, the double-pole approximation (DPA) looks as follows:

$$
\begin{aligned}
\mathscr{M}_{\mathrm{vir}, \mathrm{PA}} & = \\
& =\left.\sum_{\lambda_{1}, \lambda_{2}} \mathscr{M}_{\mathrm{virt}}^{q_{1} q_{2} \rightarrow \mathrm{w}_{1} \mathrm{w}_{2} q_{3} q_{4}} \mathscr{M}_{\mathrm{LO}}^{\mathrm{W}_{1} \rightarrow \mathrm{e}^{+} v_{\mathrm{e}} \mathscr{M}_{\mathrm{LO}}^{\mathrm{W}_{2} \rightarrow \mu^{+}} v_{\mu}}\right|_{\star} \frac{1}{K_{1} K_{2}}
\end{aligned}
$$

The shaded diagrams are examples for diagrams that are not doubly-resonant and therefore neglected in this approximation.

To restore gauge invariance an on-shell projection is used, which is symbolised by the $\star$ sign; this means that the approximated amplitude is integrated over the exact phase-space, but the momenta that are fed into the approximated matrix elements are calculated by an on-shell projection described in Ref. [16]. Also, $\star$ means that the widths of the gauge-boson in the propagators are dropped. In the resonant propagators the widths are kept and the momenta are off-shell, meaning $K_{i}=\bar{k}_{i}^{2}-M_{\mathrm{W}}^{2}+\mathrm{i} M_{\mathrm{W}} \Gamma_{\mathrm{W}}$, where $\bar{k}_{1}$ is the momentum of the first boson and $\bar{k}_{2}$ the momentum of the second one.

This procedure also modifies the IR singularities, which is taken care of by using a modified insertion operator $\mathbf{I}$,

$$
2 \operatorname{Re}\left(\mathscr{M}_{\mathrm{LO}}^{*} \mathscr{M}_{\mathrm{virt}}\right)+\left|\mathscr{M}_{\mathrm{LO}}\right|^{2} \otimes \mathbf{I} \rightarrow 2 \operatorname{Re}\left(\mathscr{M}_{\mathrm{LO}, \mathrm{PA}}^{*} \mathscr{M}_{\mathrm{virt}, \mathrm{PA}}\right)+\left|\mathscr{M}_{\mathrm{LO}, \mathrm{PA}}\right|^{2} \otimes \mathbf{I},
$$

where the subscript PA denotes the matrix elements in (double-)pole approximation.

Potentially large deviations from the exact matrix elements are known to occur in leptonic $p_{\mathrm{T}}$-distributions for di-boson production, which are due to kinematic configurations that enhance single resonant contributions [17] which are not kept in the DPA. In Ref. [17] it was argued that this can be checked for by comparing the exact LO matrix elements against the LO PA matrix elements. This comparison can be found in Fig. 2. We find only small deviations of 1-3\%, which will be even more suppressed when we use the PA only for the virtual correction.
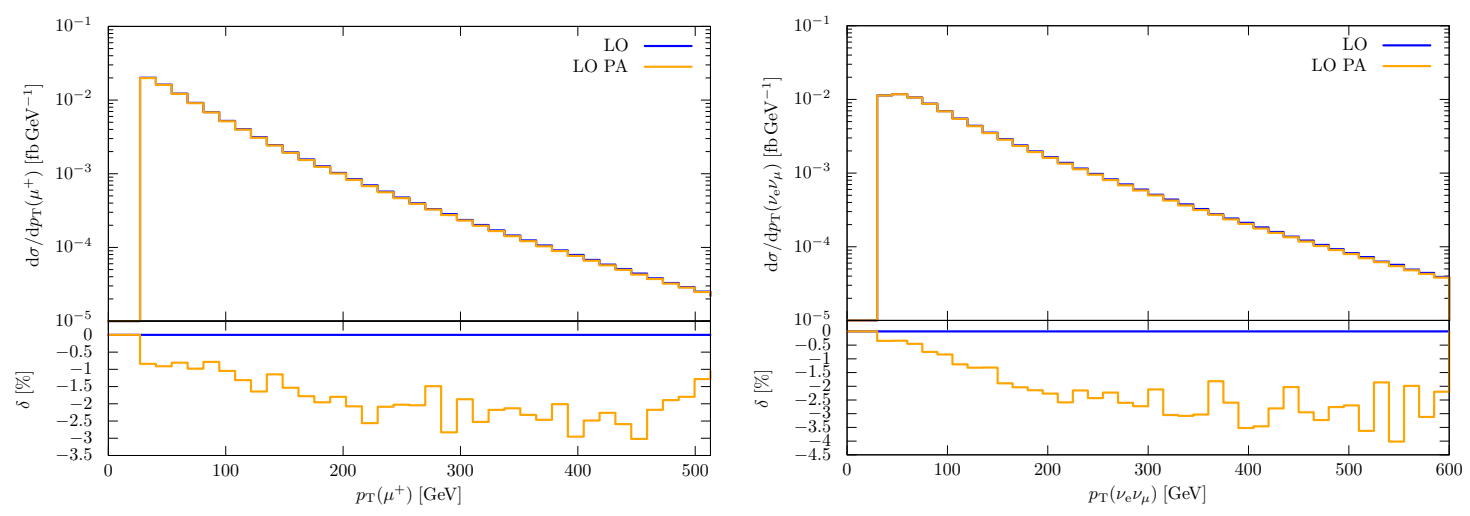

Figure 2: Leading-order comparison of exact (LO) and pole-approximated matrix elements for two observables for which large deviations have been observed for different processes. Here only very small differences are visible. 


\section{Results}

The integrated cross sections for the fiducial phase-space volume defined in Sec. 2 are list in Tab. 3. Please note again that the PDF uncertainty is given for the $90 \%$ confidence level.

\begin{tabular}{lcccccc}
\hline \multicolumn{1}{c}{ Scale } & LO & NLO & $\delta=\frac{\text { NLO-LO }}{\text { LO }}$ & \multicolumn{2}{c}{ NLO PDF unc. } & LO $M_{\mathrm{H}}=\infty$ \\
& {$[\mathrm{fb}]$} & {$[\mathrm{fb}]$} & & $\Delta_{ \pm}$ & $\Delta_{\text {sym }}$ & {$[\mathrm{fb}]$} \\
\hline static & $1.373_{-7.2 \%}^{+8.3 \%}$ & $1.193_{-3.8 \%}^{+5.8 \%}$ & $-13.1 \%$ & ${ }_{-3.2 \%}^{+3.1 \%}$ & $\pm 2.9 \%$ & 1.512 \\
dynamic & $1.222_{-6.5 \%}^{+7.3 \%}$ & $1.208_{-0.7 \%}^{+0.0 \%}$ & $-1.2 \%$ & ${ }_{-3.2 \%}^{+3.1 \%}$ & $\pm 2.9 \%$ & 1.350 \\
\hline
\end{tabular}

Figure 3: Integrated cross sections for the setup presented in Sec. 2. LO denotes the $\mathscr{O}\left(\alpha^{6}\right)$ prediction, NLO the predictions with $\mathscr{O}\left(\alpha^{6} \alpha_{\mathrm{s}}\right)$ corrections. $\Delta_{ \pm}$denotes the asymmetric PDF uncertainty and $\Delta_{\text {sym }}$ the symmetric PDF uncertainty, see Ref. [8].
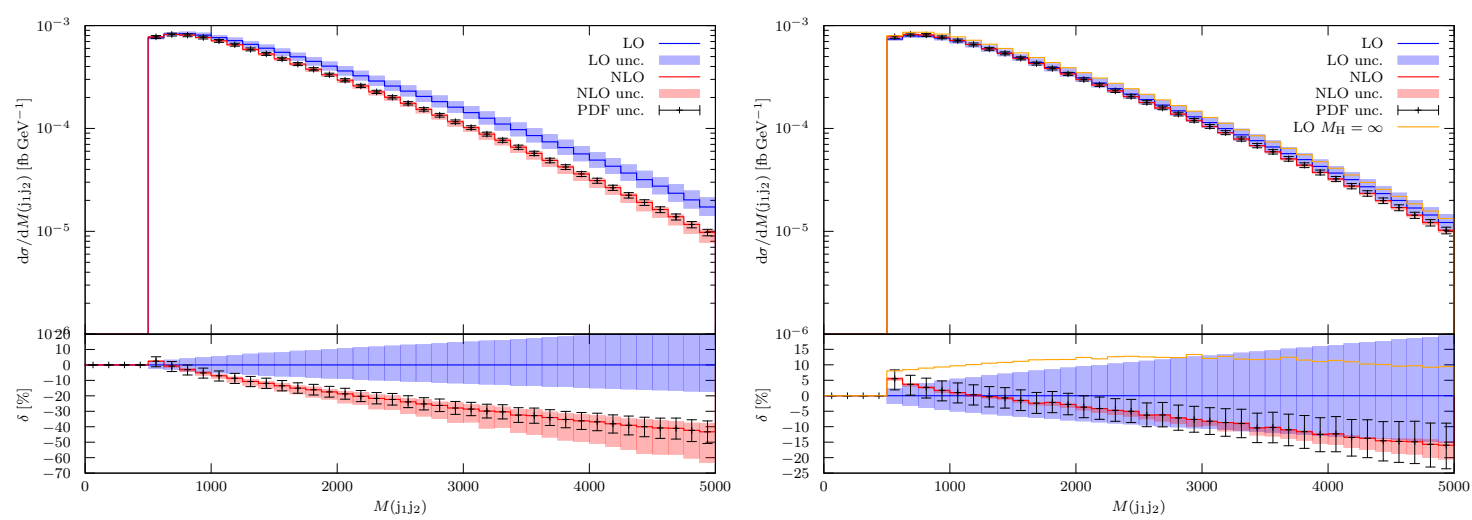

Figure 4: The invariant mass of the two tagging jets for the static scale choice (left) and the dynamic scale choice (right). PDF uncertainties are shown as black errorbars. The scale uncertainties calculated from a three-point scale variation are shown as blue (LO) and red (NLO) bands. The limit $M_{\mathrm{H}} \rightarrow \infty$ is only shown for the dynamic scale.

The results for the invariant mass of the tagging jets are shown in Fig. 4, which shows a comparison of a static scale $\mu_{\mathrm{R}}=\mu_{\mathrm{F}}=M_{\mathrm{W}}$ against our dynamic scale $\mu_{\mathrm{R}}=\mu_{\mathrm{F}}=\sqrt{p_{\mathrm{T}}^{2}\left(\mathrm{j}_{1}\right)+M_{\mathrm{W}}^{2}}$. The static scale leads to very large negative corrections where the bands of the three-point scale variation do not overlap between LO and NLO. This fact is well known [3, 4]. The observable on the right-hand side also shows that the dynamic scale choice leads to a significant reduction of the estimated scale uncertainty. For both scale choices one sees that the PDF uncertainty grows with increasing invariant mass $M\left(\mathrm{j}_{1} \mathrm{j}_{2}\right)$ of the tagging jets from $3 \%$ to $7 \%$. The effect of an absent Higgs exchange is rather flat for this observable and about $10 \%$.

Further distributions are shown in Fig. 5 which shows the rapidity difference of the tagging jets, which is increased by the NLO QCD corrections. The effect of $M_{\mathrm{H}} \rightarrow \infty$ is similar. Another distribution shown is the azimuthal angle difference of the charged leptons, which, like many other leptonic distributions, are rather unaffected by the QCD corrections. Without the Higgs the leptons are more back-to-back than with it. 

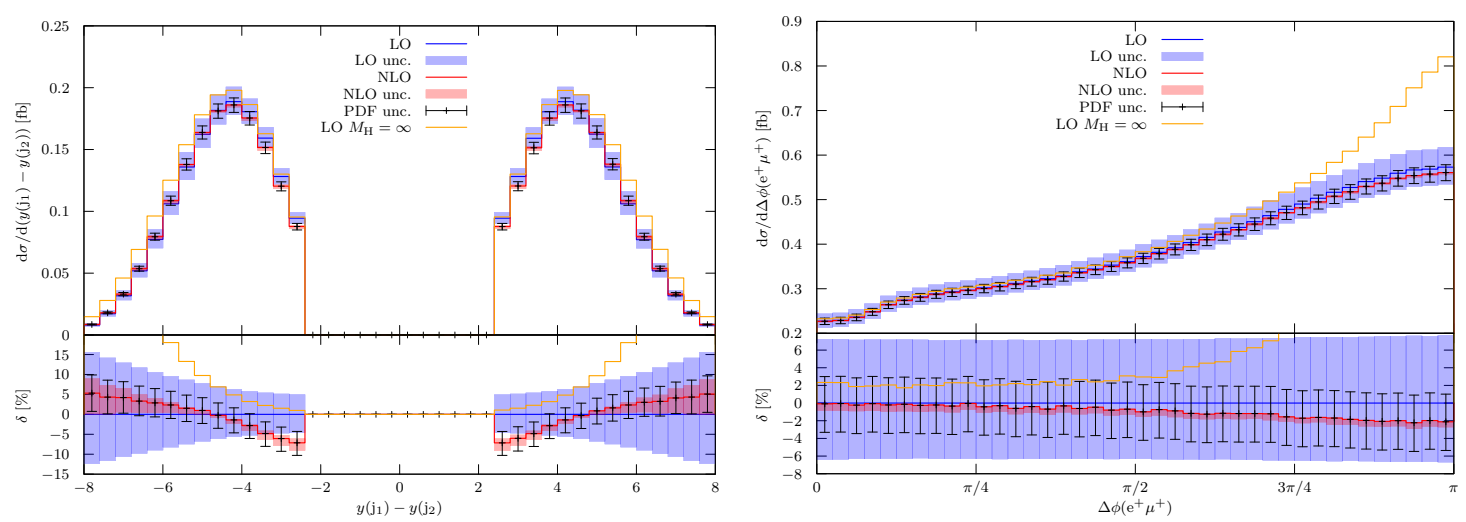

Figure 5: The rapidity gap of the tagging jets (left) and the azimuthal angle of the charged leptons (right).

\section{Acknowledgments}

The authors acknowledge support by the state of Baden-Württemberg through bwHPC and the German Research Foundation (DFG) through grant no INST 39/963-1 FUGG and grant DI 784/3. C. S. thanks Manfred Kraus for useful discussions on PDF uncertainties.

\section{References}

[1] ATLAS collaboration, G. Aad et al., Evidence for Electroweak Production of $W^{ \pm} W^{ \pm} j$ in $p p$ Collisions at $\sqrt{s}=8 \mathrm{TeV}$ with the ATLAS Detector, Phys. Rev. Lett. 113 (2014) 141803, [1405.6241].

[2] CMS collaboration, A. M. Sirunyan et al., Observation of electroweak production of same-sign W boson pairs in the two jet and two same-sign lepton final state in proton-proton collisions at $\sqrt{s}=13$ $\mathrm{TeV}, 1709.05822$.

[3] A. Denner, L. Hosekova and S. Kallweit, NLO QCD corrections to $W+W+j j$ production in vector-boson fusion at the LHC, Phys. Rev. D86 (2012) 114014, [1209. 2389 ].

[4] B. Jager, C. Oleari and D. Zeppenfeld, Next-to-leading order QCD corrections to $W+W+j j$ and $W$ W- jj production via weak-boson fusion, Phys. Rev. D80 (2009) 034022, [0907 . 0580].

[5] B. Biedermann, A. Denner and M. Pellen, Complete NLO corrections to $\mathrm{W}^{+} \mathrm{W}^{+}$scattering and its irreducible background at the LHC, JHEP 10 (2017) 124, [1708.00268].

[6] M. Cacciari, G. P. Salam and G. Soyez, The Anti-k(t) jet clustering algorithm, JHEP 04 (2008) 063, [0802.1189].

[7] S. Dulat, T.-J. Hou, J. Gao, M. Guzzi, J. Huston, P. Nadolsky et al., New parton distribution functions from a global analysis of quantum chromodynamics, Phys. Rev. D93 (2016) 033006, [1506. 07443 ].

[8] J. Pumplin, D. Stump, R. Brock, D. Casey, J. Huston, J. Kalk et al., Uncertainties of predictions from parton distribution functions. 2. The Hessian method, Phys. Rev. D65 (2001) 014013, [hep-ph/0101032].

[9] S. Dittmaier and M. Roth, LUSIFER: A LUcid approach to six FERmion production, Nucl. Phys. B642 (2002) 307-343, [hep-ph/ 0206070$].$ 
[10] A. Denner, S. Dittmaier and L. Hofer, Collier: a fortran-based Complex One-Loop LIbrary in Extended Regularizations, Comput. Phys. Commun. 212 (2017) 220-238, [1604 . 06792 ].

[11] A. Denner and S. Dittmaier, Reduction of one loop tensor five point integrals, Nucl. Phys. B658 (2003) 175-202, [hep-ph/0212259].

[12] A. Denner and S. Dittmaier, Reduction schemes for one-loop tensor integrals, Nucl. Phys. B734 (2006) 62-115, [hep-ph/0509141].

[13] A. Denner and S. Dittmaier, Scalar one-loop 4-point integrals, Nucl. Phys. B844 (2011) 199-242, [1005.2076].

[14] T. Gleisberg, S. Hoeche, F. Krauss, M. Schonherr, S. Schumann, F. Siegert et al., Event generation with SHERPA 1.1, JHEP 02 (2009) 007, [0811 . 4622].

[15] F. Cascioli, P. Maierhofer and S. Pozzorini, Scattering Amplitudes with Open Loops, Phys. Rev. Lett. 108 (2012) 111601, [1111.5206].

[16] S. Dittmaier and C. Schwan, Non-factorizable photonic corrections to resonant production and decay of many unstable particles, Eur. Phys. J. C76 (2016) 144, [1511.01698].

[17] B. Biedermann, M. Billoni, A. Denner, S. Dittmaier, L. Hofer, B. Jäger et al., Next-to-leading-order electroweak corrections to $p p \rightarrow W^{+} W^{-} \rightarrow 4$ leptons at the LHC, JHEP 06 (2016) 065 , [1605.03419]. 